\title{
The associations of active and passive social media use with well-being: A critical scoping review
}

new media \& society

I-20

(C) The Author(s) 2021

Article reuse guidelines: sagepub.com/journals-permissions DOI: $10.1|177 /| 46 \mid 4448211065425$ journals.sagepub.com/home/nms

\author{
Patti M ValkenburgiD, \\ Irene I van Driel and Ine Beyens \\ University of Amsterdam, The Netherlands
}

\begin{abstract}
A recurring claim in the literature is that active social media use (ASMU) leads to increases in well-being, whereas passive social media use (PSMU) leads to decreases in well-being. The aim of this review was to investigate the validity of this claim by comparing the operationalizations and results of studies into the association of ASMU and PSMU with well-being (e.g. happiness) and ill-being (e.g. depressive symptoms). We found 40 survey-based studies, which utilized a hodgepodge of 36 operationalizations of ASMU and PSMU and which yielded 172 associations of ASMU and/or PSMU with well-/ill-being. Most studies did not support the hypothesized associations of ASMU and PSMU with well-/ill-being. Time spent on ASMU and PSMU may be too coarse to lead to meaningful associations with well-/ill-being. Therefore, future studies should take characteristics of the content of social media (e.g. the valence), its senders (e.g. preexisting mood), and receivers (e.g. differential susceptibility) into account.
\end{abstract}

\section{Keywords}

Depression, Facebook, Instagram, media effects, social media, well-being

The past 3 years have yielded more than four dozen meta-analyses and reviews on the associations of social media use (SMU) with indicators of well-being, such as life satisfaction and happiness (Valkenburg, 2022). In some of these meta-analyses and reviews, two types of SMU have been conceptualized, active and passive. Active social media use

\footnotetext{
Corresponding author:

Patti M Valkenburg, Amsterdam School of Communication Research, University of Amsterdam, Spui 2I, Amsterdam 1012 CX, The Netherlands.

Email: p.m.valkenburg@uva.nl
} 
(ASMU) entails "targeted one-on-one exchanges," such as sending private messages or "broadcasting" (e.g. posting a status update), whereas passive social media use (PSMU) refers to "monitoring the online life of other users without engaging in direct exchanges with them" (e.g. scrolling or looking at other users" profiles, Verduyn et al., 2020: 3). One prominent hypothesis in these reviews is that ASMU leads to positive effects on wellbeing, because it elicits support and positive feedback, which can subsequently increase well-being. Another hypothesis is that PSMU leads to declines in well-being, because it induces upward social comparison and envy (Verduyn et al., 2017).

Despite the prominence of the ASMU and PSMU hypotheses in the literature, several recent studies have criticized the active-passive dichotomy in social media (SM) research. Some of these critiques relate to the lack of valid instruments to measure ASMU and PSMU (Trifiro and Gerson, 2019). Others argue that the active-passive dichotomy needs refinement, because some types of ASMU could lead to decreases rather than increases in well-being (Kross et al., 2021) or because PSMU cannot only lead to envy but also to inspiration and positive effects on well-being (Meier et al., 2020). And yet others argue that the concept "passive" SMU challenges long-standing communication theories that consider the reception of media messages as active, in the sense that recipients have autonomy over the way they select, process, and interpret media messages (Valkenburg et al., 2022).

The overall aim of the current scoping review is to respond to these criticisms by systematically evaluating the latest empirical basis of the hypothesized associations of ASMU and PSMU with well-being. These associations have been examined in three recent meta-analyses (Hancock et al., 2019; Liu et al., 2019; Yin et al., 2019), which together yielded disagreeing pooled effect sizes. Whereas Hancock et al. (2019) reported a positive association of ASMU with well-being that is consistent with the ASMU hypothesis, both Yin et al. (2019) and Liu et al. (2019) found associations that contradict this hypothesis. Conversely, whereas Liu et al. revealed a significant negative association of PSMU with well-being in support of the PSMU hypothesis, both Hancock et al. and Yin et al. reported associations of PSMU that disconfirm this hypothesis.

The meta-analyses not only revealed disagreeing pooled effect sizes of the associations of ASMU and PSMU with well-being, but they also reported considerable heterogeneity in these pooled effect sizes (Liu et al., 2019; Yin et al., 2019), which indicates that differences in the reported effect sizes across the included studies cannot be explained by chance alone (Melsen et al., 2014). Considerable heterogeneity in meta-analyses may be due to methodological differences across the included studies (Melsen et al., 2014), for example, in study designs, operationalizations, and outcomes of ASMU and PSMU. However, when a body of literature exhibits a heterogeneous nature, it is not amenable to a meta-analysis. In such cases, a scoping review is the most feasible alternative (Peters et al., 2015). A scoping review is, therefore, the purpose of the present study.

In our scoping review, we try to explain the heterogeneity in findings across studies by coding the operationalizations and types of outcomes of ASMU and PSMU of each individual study to critically investigate potential differences in results across studies. Our review included studies that appeared from January 2017 to October 2021, because earlier meta-analyses already discussed studies up to January 2017. An up-to-date research synthesis is important because in recent years, several sophisticated longitudinal and 
experimental research designs have been employed, which may provide renewed insights into the assumed associations of ASMU and PSMU with well-being.

\section{Well-being versus ill-being}

Recent empirical studies into the effects of ASMU and PSMU have investigated not only a range of indicators of well-being (e.g. life satisfaction) but also a range of indicators of ill-being (e.g. depression, Ryff et al., 2006). This bipartite conceptualization is in part the result of historical developments. Prior to the 1950s, well-being was predominantly defined as ill-being (Greenspoon and Saklofske, 2001). With the rise of positive psychology in the 1980s (e.g. Diener, 1984) came an emphasis on subjective well-being, which is the experience of happiness, positive affect, and life satisfaction. However, it is now well understood that well-being is not simply the flip side of ill-being, meaning that the absence of ill-being does not guarantee a high well-being and vice versa (e.g. Ryff et al., 2006).

Over the past decades, there has been a growing understanding that well-being and ill-being should be conceptualized as two distinct continuums (Greenspoon and Saklofske, 2001; Meier and Reinecke, 2020). Therefore, we separately review the associations of ASMU and PSMU with each of the indicators of well-being and ill-being and investigate whether these associations differ for well-/ill-being outcomes. We focus on three indicators of well-being: happiness, life satisfaction, and positive affect, as well as three indicators of ill-being: depressive symptoms/mood, depression, and negative affect. We do not consider other indicators of ill-being, such as anxiety, because these are less frequently investigated than depression/depressive symptoms (e.g. Keles et al., 2020; Liu et al., 2019) and thus more difficult to compare across studies. Because the type of outcome may be an explanation for the heterogeneity in effect sizes across studies, our first research question asks,

$R Q 1$. Are the associations of ASMU and PSMU with well-being outcomes different from those with ill-being outcomes?

\section{Private and public ASMU and PSMU}

Burke et al. (2010) were the first to suggest that Facebook activities "can be dichotomized into active and passive forms of usage" (Verduyn et al., 2015: 480). Like Burke et al., the majority of the "early" studies into the associations of ASMU and/or PSMU have focused on Facebook (e.g. Deters and Mehl, 2012; Wenninger et al., 2014). In these studies, ASMU and PSMU were operationalized as public ASMU (i.e. posting status updates and commenting on posts) and public PSMU (i.e. scrolling through news feeds and viewing posts). However, contemporary SM users at least as often use SM platforms for private SMU (Waterloo et al., 2018). Private SMU typically entails the synchronous, one-to-one or small group interactions between known communication partners (e.g. chatting via Facebook or WhatsApp).

Private ASMU and PSMU differ from their public counterparts in that they are more commonly used, more synchronous, and more intimate (Bazarova et al., 2015; van Driel 
et al., 2019; Waterloo et al., 2018). This difference has three implications. First, private ASMU occurs almost twice as frequently as public ASMU (Faelens et al., 2019; Frison and Eggermont, 2020), which may result in stronger associations with well-being and/or ill-being. Second, in synchronous private SM interactions, ASMU and PSMU may be more difficult to disentangle than in their public, more asynchronous equivalents, which would, in turn, imply that the intercorrelations of private ASMU and PSMU will be stronger than those of public ASMU and PSMU. Third, because of the differences in intimacy between public and private forms of SMU, it is conceivable that the associations of private ASMU and PSMU with well-/ill-being deviate from those of their public counterparts. Altogether, because differences in the frequency and nature of private and public ASMU and PMSU may have contributed to the heterogeneity in findings across studies, we investigate the following research questions:

$R Q 2$. Are the frequencies of public and private ASMU different from those of public and private PSMU?

$R Q 3$. Are the intercorrelations between private ASMU and PSMU stronger than those between public ASMU and PSMU?

$R Q 4$. Are the associations of private ASMU and PSMU with well-being and ill-being different from those of public ASMU and PSMU?

\section{Different designs, different effects}

With the recent proliferation of studies into the associations of ASMU and PSMU with well-being and ill-being came a diversity of research designs, such as longitudinal, experience sampling method (ESM), and experimental designs. This wider database of studies offers the opportunity to investigate whether the hypothesized associations of ASMU and PSMU also hold in more powerful designs, such as longitudinal studies. In addition, new methods of analysis have been gaining prominence that are able to disentangle within-person effects of SMU from between-person associations, for example, as applied in random-intercept cross-lagged panel (Puukko et al., 2020) and multi-level models (Beyens et al., 2020).

Studies that focus on within-person effects investigate to what extent SMU leads to changes in well-being within a person compared to this person's average well-being. Within-person methods are more valid to investigate media effects than traditional between-person methods (for further discussion, see Valkenburg et al., 2022). After all, a media effect takes place within persons and not between persons. A media effect is an intra-individual change within a person due to this person's media use (Valkenburg et al., 2016), and such intra-individual changes should be investigated with within-person rather than between-person methods. Therefore, we investigate the following question:

RQ5. Are the associations of private and public ASMU and PSMU with well-being and ill-being found in cross-sectional survey studies different from the between-person and within-person associations found in longitudinal studies? 


\section{Method}

\section{Search strategy}

We searched for journal articles and preprints that were published in the period from January 2017 to October 2021. We started with Web of Science, using the following search terms: Topic (TS) $=$ (["social media" or SNS or "social network" or Facebook or Instagram or WeChat] and [active or passive or brows* or post*] and [effect or association* or relation*] and ["well-being" or "mental health" or depress* or happiness or "lifesatisfaction"]). This search yielded 749 hits.

In a next step, an additional Google Scholar search was conducted, first to find potential additional publications and second because, unlike Web of Science, the Google Scholar database includes preprints. Since Google Scholar lists endless more or less relevant preprints and publications, we ended our screening after page 20 of the results. All hits of Web of Science and Google Scholar were screened by the first two authors. Relevant studies were selected with $100 \%$ agreement after discussion.

\section{Exclusion criteria}

We excluded studies on active and passive general screen time (e.g. Kim et al., 2020). We also excluded studies focusing on other indicators of ill-being than depressive symptoms/mood depression, and negative affect, such as anxiety and stress, as well as potential precursors of well-/ill-being, such as self-esteem, loneliness, and social capital. Several of the eligible studies focused on additional outcomes than the ones included in our search, such as self-esteem (Burnell et al., 2020) or anxiety (Thorisdottir et al., 2019). Moreover, some studies investigated the indirect effects of ASMU or PSMU on wellbeing, for example, via friendship support (Frison and Eggermont, 2020), social comparison (Meier et al., 2020), or concern about feedback (Yang, 2020). To enhance the comparability across the studies, we included all these studies in our review but only report the direct associations of ASMU and PSMU with the selected well-/ill-being outcomes.

Finally, some of the included studies were based on the same samples. Specifically, the results of Wang et al. (2018), Wang et al. (2019), and Zhang et al. (2020) were based on the same sample. Because these studies used different outcomes (e.g. negative emotions vs depression), we included them as separate studies in our review. This criterion did not apply to Aalbers et al. (2019) versus Rodriguez et al. (2021), and Beyens et al. (2021) versus Valkenburg et al. (2022), which both employed the same predictors and outcomes. In these cases, we only included the original study, that is, the study that was published first.

\section{Coding strategy}

Each of the studies included in this review was coded for 16 different types of information by the first two authors. Potential disagreements, which hardly occurred, were solved by discussion: 
1. Type of study (i.e. cross-sectional, longitudinal, experiment, ESM study)

2. Sample size

3. Age of sample (e.g. adult vs adolescent)

4. Type of sample (e.g. general sample vs university students vs Facebook users)

5. Country of origin of study

6. Investigated platform(s) (e.g. Facebook, unspecified/general)

7. Investigated well-being and ill-being outcome

8. Operationalization of ASMU and/or PSMU (number and wording of items)

9. Number and type of response options for ASMU and PSMU (e.g. 5-point Likerttype scale, 7-point frequency scale, direct time estimate)

10. Public ASMU (i.e. posting content, photos, status updates) or PSMU (browsing or reading profiles), private ASMU (sending messages) or PSMU (reading messages)

11. Potential other specifics of ASMU and/or PSMU (e.g. valence, communication partner)

12. Between- and/or within-person association of ASMU and/or PSMU with wellbeing/ill-being

13. Average frequency/duration of usage of different types of ASMU and/or PSMU

14. Average frequency/duration of ASMU and/or PSMU as indicated in the response options (i.e. once a month, several times a week)

15. Correlations between ASMU and PSMU

16. Prevalence ratios of public ASMU versus public PSMU, and private ASMU versus private PSMU.

\section{Inference criteria and effect sizes of interest}

Because the sample sizes, and thus the power of the included studies, deviated considerably across studies, we relied on effect sizes rather than significance levels. Following Gignac and Szodorai (2016), a cross-sectional effect size ranging from $r=-.10$ to $r=$ +.10 was interpreted as "non-existent to very small," and all cross-sectional effect sizes beyond this range as negative or positive. Longitudinal between-person and within-person associations ranging from -.05 to +.05 were interpreted as "non-existent to very small," and all associations beyond this range as negative or positive (Adachi and Willoughby, 2015). This means that a cross-sectional effect size of $r \geqslant+.10$ and a longitudinal effect size of $\beta \geqslant+.05$ of ASMU with well-being outcomes or a cross-sectional association of $r \leqslant-.10$ or a longitudinal effect size of $\beta \leqslant-.05$ of ASMU with ill-being outcomes provided support for the ASMU hypothesis. Conversely, a cross-sectional effect size of $r \leqslant-.10$ and a longitudinal effect size of $\beta \leqslant-.05$ of PSMU with well-being outcomes or a cross-sectional association of $r \geqslant+.10$ or a longitudinal effect size of $\beta \geqslant$ +.05 of PSMU with ill-being outcomes provided support for the PSMU hypothesis.

\section{Results}

\section{Designs and samples of the included studies}

A total of 45 studies published from January 2017 to October 2021 met our inclusion criteria, of which one study contained a survey and an experiment (Hanley et al., 2019), 
resulting in a total of 40 survey-based studies (cross-sectional, longitudinal, and ESM studies) and six experiments. Three studies were preprints (Beyens et al., 2021; Rodriguez et al., 2021; Valkenburg et al., 2022) but these were published before this review study was finished. Of all 45 studies, 16 were conducted in Europe, 2 in the Middle East, 12 in the Far East, 10 in America, 3 in Australia, and 2 in multiple English-speaking countries. Ten studies focused on (pre)adolescents and the remaining 35 on adults.

Because our aim was to compare operationalizations of ASMU and PSMU across studies, this review focused on the 40 survey-based studies. The experiments were used to complement the results of the survey-based studies. Most survey-based studies involved cross-sectional studies $(n=27)$, followed by ESM studies $(n=7)$, and longitudinal studies $(n=6)$. An overview of the designs and samples of the studies is provided in Supplemental Appendix A.

\section{Investigated SM platforms}

Of the 40 survey-based studies, 22 (55\%) focused on one platform, mostly Facebook (14); 10 studies (25\%) did not specify specific platforms; and 8 (20\%) investigated more than one platform.

\section{Incomplete information in the studies}

Eighteen out of the 40 survey-based studies lacked one or more types of information necessary to compare their results. In fact, 16 studies lacked information about the operationalization of ASMU and/or PSMU (e.g. specific items and response options), and 6 about one or more of the results (e.g. intercorrelations of ASMU and PSMU, direct associations of ASMU and PSMU with well-/ill-being). Except for one study (of which the results are not included), all authors were willing and able to provide the missing information upon request. Because some information in the Supplemental Appendices of this article cannot be found in the published papers, we have marked the studies for which the authors provided additional information in these Supplemental Appendices.

\section{Operationalizations of ASMU and PSMU}

Supplemental Appendix A lists the operationalizations of ASMU and PSMU employed in the 40 survey-based studies. Of these studies, 36 (90\%) used a unique operationalization of ASMU and/or PSMU. Together, these studies used 29 variations of response scales to measure ASMU and PSMU.

\section{Differential associations of ASMU and PSMU with well-being versus ill- being}

The ASMU hypothesis argues that ASMU (e.g. posting, sending messages) increases well-being and/or decreases ill-being, because it may elicit likes, support, and positive feedback from fellow SM users. Conversely, the PSMU hypothesis claims that PSMU (e.g. browsing, reading messages) leads to decreases in well-being and/or increases in 
ill-being, because it induces upward social comparison and envy among these browsers/ readers. However, because well-being and ill-being are not opposite ends of a bipolar scale, and should be conceptualized as two separate continuums, our first research question asked whether the associations of ASMU and PSMU would differ for well-being and ill-being outcomes.

Our results revealed considerable heterogeneity in both the reported associations of ASMU and PSMU with well-being and ill-being outcomes. For example, as Supplemental Appendix B shows, the associations of ASMU with life satisfaction ranged from $r=-.08$ (Masciantonio et al., 2021) to $r=+.26$ (Lian et al., 2020), while those with depression/depressive symptoms ranged from $r=-.07$ (Brailovskaia and Margraf, 2019) to $r=+.17$ (Frison and Eggermont, 2020). However, despite this considerable heterogeneity, the ASMU hypothesis received stronger support for the well-being than for the ill-being outcomes. As Table 1 shows, of the 56 cross-sectional and longitudinal associations of ASMU with well-being, only 22 (39\%) were in line with the ASMU hypothesis. Remarkably though, of all 36 cross-sectional and longitudinal associations of ASMU with ill-being, none $(0 \%)$ were in line with the ASMU hypothesis.

Conversely, the PSMU hypothesis received stronger support for the ill-being than well-being outcomes: As Table 1 shows, of all 34 cross-sectional and longitudinal illbeing associations, 15 (44\%) were in line with the PSMU hypothesis. Finally, of all 46 well-being associations, 7 (15\%) provided support for the PSMU hypothesis. However, despite these reversed results for well-being and ill-being outcomes, for both outcomes, only a minority of the associations supported the ASMU and PSMU hypotheses.

\section{Frequencies of public versus private ASMU and PSMU}

Our second research question addressed the relative frequency of public and private ASMU and PSMU. To investigate this research question, we coded for all operationalizations of ASMU and PSMU whether their items pertained to public or private ASMU and/or PSMU. During this coding process, we discovered that 12 operationalizations $(30 \%)$ combined items measuring private ASMU (sending private messages) or private PSMU (reading private messages) with items measuring public ASMU or public PSMU. However, as argued, private SMU may differ from public SMU both in its frequency and nature (e.g. intimacy, reciprocity) and thus its effects. Therefore, to avoid this "private-public confound," we investigated these operationalizations as a separate category, which we labeled as "mixed ASMU" or "mixed PSMU." Because of space restrictions, we included the results of these operationalizations in Supplemental Appendices A and B but do not summarize them in the Results section.

In addition, in nine survey-based studies, private ASMU was confounded with private interactive SMU (i.e. chatting). However, chatting refers to the dynamic giveand-take interactions between two or small groups of SM users, which, intrinsically, consist of both ASMU and PSMU. To investigate the hypothesis that ASMU leads to opposite effects on well-being than PSMU, combining ASMU and PSMU into the 
Table I. Percentages of associations that provided support for the ASMU and PSMU hypotheses.

\begin{tabular}{|c|c|c|}
\hline Type of association & $\begin{array}{l}\text { Support for ASMU } \\
\text { hypothesis }{ }^{\mathrm{a}} \\
\%(n)^{\mathrm{b}}\end{array}$ & $\begin{array}{l}\text { Support for PSMU } \\
\text { hypothesis }{ }^{\mathrm{a}} \\
\%(n)^{\mathrm{b}}\end{array}$ \\
\hline \multicolumn{3}{|l|}{ Research question I } \\
\hline All associations with well-being & $39 \%(22 / 56)$ & $15 \%(7 / 46)$ \\
\hline All associations with ill-being & $0 \%(0 / 36)$ & $44 \%(15 / 34)$ \\
\hline \multicolumn{3}{|l|}{ Research question 4} \\
\hline All public associations with well-/ill-being & $21 \%(12 / 58)$ & $26 \%(18 / 68)$ \\
\hline All private associations with well-/ill-being & $10 \%(1 / 10)$ & $20 \%(1 / 5)$ \\
\hline \multicolumn{3}{|l|}{ Research question 5} \\
\hline $\begin{array}{l}\text { Cross-sectional public associations with } \\
\text { well-/ill-being }\end{array}$ & $18 \%(9 / 50)$ & $27 \%(16 / 59)$ \\
\hline $\begin{array}{l}\text { Longitudinal public associations with well-/ } \\
\text { ill-being }\end{array}$ & $38 \%(3 / 8)$ & $40 \%(2 / 5)$ \\
\hline $\begin{array}{l}\text { Cross-sectional private associations with } \\
\text { well-/ill-being }\end{array}$ & $17 \%(1 / 6)$ & $33 \%(1 / 3)$ \\
\hline $\begin{array}{l}\text { Longitudinal private associations with well-/ } \\
\text { ill-being }\end{array}$ & $0 \%(0 / 4)$ & $0 \%(0 / 2)$ \\
\hline
\end{tabular}

anference criteria: A cross-sectional effect size of $r \geqslant+.10 / r \leqslant-10$ and a longitudinal effect size of $\beta \geqslant$ $+.05 / \beta \leqslant-.05$ with well-being/ill-being provided support for the ASMU hypothesis. Conversely, a crosssectional effect size of $r \leqslant-.10 / r \geqslant+.10$ and a longitudinal effect size of $\beta \leqslant-.05 / \beta \geqslant+.05$ with wellbeing/ill-being provided support for the PSMU hypothesis.

bNumber of associations that supported the hypothesis/total number of associations.

same items may render a test of this hypothesis invalid. Because seven of these confounded operationalizations were already marked as mixed ASMU, their results are only included in Supplemental Appendix B and not discussed in the text. Because the remaining two operationalizations measured private ASMU and included only one item referring to chatting, we coded them as private ASMU (see Supplemental Appendix A).

Of the 15 survey-based studies that focused on both public ASMU and public PSMU, three found that the mean of ASMU was about $10 \%$ higher than the mean of PSMU. These studies were all based on the same sample of Chinese students and investigated the popular platform Qzone (Wang et al., 2018, 2019; Zhang et al., 2020). For one study, the measures did not allow for such a comparison because it used a time scale for ASMU and a frequency scale for PSMU. Across the remaining 11 studies, the mean of public PSMU was on average 133\% higher (ratio: 2.33) than that for public ASMU (see Supplemental Appendix B). Finally, three studies compared the frequencies of private ASMU and private PSMU. These studies showed that the mean of private PSMU was only $11 \%$ higher (ratio: 1.11) than that of private ASMU. In all, we found significantly higher usage frequencies for public PSMU than for public ASMU, while the usage frequencies of private PSMU and ASMU differed hardly at all. 


\section{Intercorrelations between public and private ASMU and PSMU}

Our third research question asked whether the correlations between private ASMU and PSMU would be higher than those between public ASMU and PSMU. As Supplemental Appendix B shows, most of the intercorrelations of public ASMU and PSMU centered between $r=.30$ and $r=.55$, with three outliers $(r=.12$, Jarman et al., 2021; $r=-.39$, Masciantonio et al., 2021; $r=.08$, Wenninger et al., 2019). The three studies that reported on private ASMU and PSMU yielded considerably higher intercorrelations, ranging from $r=.75$ to $r=.99$. In all, these results indicate that the correlations between private ASMU and PSMU were significantly higher than for their public counterparts, which underscores the synchronous and dynamic character of private SMU.

\section{Associations of public and private ASMU and PSMU with well-/ill-being}

The ASMU hypothesis does not distinguish between public and private SMU and thus predicts that both types of ASMU would lead to increases in well-being and decreases in ill-being. Conversely, the PSMU hypothesis predicts that both public and private PSMU would lead to decreases in well-being and increases in ill-being. However, since private SMU differs in several respects from public SMU, we believe there is reason to assume that the effects on well-/ill-being differ for private and public ASMU and PSMU. Therefore, our fourth research question asked whether private ASMU and PSMU would lead to different associations with well-/ill-being than public ASMU and PSMU would. In addition, our fifth research question asked to what extent the associations of private and public ASMU and PSMU with well-/ill-being found in the cross-sectional studies would hold for the between-person and within-person associations found in longitudinal studies.

Public ASMU. Of the 50 cross-sectional associations, $18 \%(n=9)$ confirmed the public ASMU hypothesis (see Table 1). The remaining $82 \%$ associations did not support the ASMU hypothesis. Of the four between-person associations found in longitudinal studies, only one (25\%) was consistent with the public ASMU hypothesis. Finally, as for the four longitudinal within-person associations, two (50\%) were supportive of the public ASMU hypothesis. The remaining two were non-existent to very small and thus unsupportive of this hypothesis.

Private ASMU. Of the six cross-sectional associations, only one (17\%) confirmed the private ASMU hypothesis. In addition, none $(0 \%)$ of the four between- and within-person associations found in longitudinal studies provided support for the private ASMU hypothesis.

Public PSMU. Of the 59 cross-sectional associations, $16(27 \%)$ confirmed the public PSMU hypothesis, whereas $73 \%(n=43)$ did not confirm this hypothesis (see Table 1$)$. Of the five longitudinal between-person associations, two (40\%) provided support for the PSMU hypothesis, whereas three $(60 \%)$ did not. Finally, all four within-person associations were too small $(-.04 \leqslant \beta \leqslant .01)$ to provide support for the public PSMU hypothesis. 
In addition to the studies that investigated general time spent on PSMU, several studies investigated how specific sub-types of PSMU would influence well-/ill-being. These studies found that public PSMU increased well-being/decreased ill-being only or particularly when (1) PSMU involved non-social profiles rather than social profiles (Alfasi, 2019; Chen et al., 2019), (2) PSMU pertained to one's own profile rather than those of others (Burnell et al., 2020; Yuen et al., 2019), (3) the posts were positive (Choi and Kim, 2021), (4) enjoyable (Valkenburg et al., 2022), or (5) inspiring (Meier et al., 2020).

Private PSMU. Finally, as Table 1 shows, only one (33\%) out of three cross-sectional associations and none of the two within-person associations confirmed the PSMU hypothesis.

\section{Discussion}

The aim of this scoping review was to investigate the validity of the ASMU and PSMU hypotheses. These hypotheses argue that ASMU (e.g. posting, sending messages) leads to increases in well-being/decreases in ill-being, because it elicits likes and support, whereas PSMU (e.g. browsing, reading messages) results in decreases in well-being/ increases in ill-being because it induces social comparison and envy. We coded the operationalizations and results of 40 survey-based studies to investigate whether and how different types of ASMU and PSMU lead to differences in well-being and/or ill-being.

Like the three meta-analyses (Liu et al., 2019; Yin et al., 2019), our review yielded limited support for both the ASMU and PSMU hypothesis. In fact, 79\% of all associations disconfirmed the public ASMU hypothesis, whereas $74 \%$ of all associations refuted the public PSMU hypothesis. Likewise, 90\% of all associations refuted the private ASMU hypothesis and $80 \%$ the private PSMU hypothesis. Finally, like the meta-analyses, we found considerable heterogeneity in the effect sizes of ASMU and PSMU. In the remainder of this article, we discuss seven potential explanations for this heterogeneity, accompanied by seven suggestions for future research.

\section{Explanation I: well-being is not the flip side of ill-being}

Overall, the ASMU hypothesis received stronger support for the well-being than illbeing outcomes, whereas the PSMU hypothesis was more strongly supported by the illbeing than well-being outcomes (RQ1, see Table 1). This result confirms earlier accounts that well-being is not simply the flip side of ill-being and vice versa and that both wellbeing and ill-being should be conceptualized as two separate continuums (Meier and Reinecke, 2020). Future research should avoid the "well-being/ill-being confound" by either clearly justifying their choice of outcome or by including and comparing the associations of ASMU and PSMU with both well-being and ill-being outcomes.

\section{Explanation 2: public PSMU occurs twice as often as public ASMU}

Our analysis revealed that the means for public PSMU were more than twice as high as those for public ASMU (RQ2). This difference in frequency implies that respondents' 
total public SMU consisted for about $69 \%$ of PSMU and for about $31 \%$ of ASMU. Our result has been confirmed by Erliksson et al. (2020), who found that PSMU explained $81 \%$ of the variance in total SMU, whereas ASMU explained $49 \%$ of this variance. This skewed ratio indicates that the two types of use do not only differ in their active and passive nature, but also in their frequency. Activities that occur more frequently could have a greater impact, meaning that the effects of public PSMU in some studies may have been stronger than those of ASMU, not because of the passive nature of PSMU but because of its higher frequency.

The relative infrequent public ASMU may be particularly problematic when studies focus on one platform, which 55\% of the included studies did. In such studies, public ASMU may turn out to be unrealistically low. For example, in some studies, the frequency of active Facebook use was around "1 to 3 times a month" (Faelens et al., 2019; Frison and Eggermont, 2020). However, people, adolescents and adults alike, typically use three to five platforms in a complementary way (Waterloo et al., 2018), to interact with their friends or to present themselves to a wider audience. Future research should therefore try to minimize the "frequency confound" by focusing on more than one SM.

\section{Explanation 3: private SMU differs from public SMU}

Our results yielded considerably stronger intercorrelations between private ASMU and PSMU than between their public counterparts (RQ3). In addition, our results revealed stronger support for the public ASMU and PSMU hypotheses than for the private ASMU and PSMU hypotheses (RQ4). These results indicate, first, that private ASMU and PSMU are more synchronous and more difficult to disentangle than their public equivalents, and second, that the associations of private ASMU and PSMU with well-being and ill-being differ from those of public ASMU and PSMU.

Yet, our analysis revealed that $28 \%$ of the included studies combined private and public items in their operationalizations of ASMU and/or PSMU. Given that the associations of private ASMU and PSMU differ from their public equivalents in frequency and synchronicity, as well as in their associations with well-being and ill-being, the "privatepublic confound" is a third factor that may have caused the heterogeneity across studies. This confound should, therefore, be avoided in future research.

\section{Explanation 4: ASMU and PSMU are difficult to disentangle empirically}

Although private ASMU and PSMU are empirically more difficult to disentangle than their public counterparts, this difficulty also applies to some specific types of public ASMU and PSMU. A striking example involves the operationalization of "likes" and "comments". One could argue that liking and/or commenting on someone's post is ASMU, because it involves sending a fellow user a like, support, or a compliment. However, dichotomizing likes and comments is less straightforward than it may seem at first sight. In some included operationalizations, likes and/or comments were indeed a priori conceptualized as ASMU (Brailovskaia and Margraf, 2019; Masciantonio et al., 2021; Pang, 2021), but in an equal number of other operationalizations, likes and/or comments were conceptualized as PSMU (Hanna et al., 2017; Lin et al., 2021; Tosun and Kaşdarma, 2020). 
Remarkably though, in the operationalizations of ASMU and PSMU that were based on factor analyses, likes and/or commenting loaded about equally on both the ASMU and PSMU factors (Escobar-Viera et al., 2018; Hanley et al., 2019; Nisar et al., 2019). Apparently, likes and comments fail to be empirically dichotomized in ASMU and PSMU (Bayer et al., 2020; Carr et al., 2016). However, likes and comments are among the most common activities on SM platforms, and it may be indicative of the invalidity of the active-passive dichotomy that such behaviors defy categorization. Our review revealed that $90 \%$ of the included studies created their own idiosyncratic operationalization of ASMU and PSMU. Many of these operationalizations were not or only partly shared in the articles. Future researchers may turn to Supplemental Appendix A and B of our review to decide whether, how, and to what extent a dichotomous conceptualization of ASMU and PSMU will further extend the SM effects literature.

\section{Explanation 5: ASMU and PSMU are difficult to disentangle conceptually}

Disentangling the effects of ASMU and PSMU is not only empirically problematic but also conceptually. When deconstructing the ASMU hypothesis, it becomes clear that the positive effect of ASMU on well-being is attributed to its potential to elicit likes and support, which in turn stimulates the well-being of the active user (Verduyn et al., 2017). However, reading the support, positive feedback, or likes is, essentially, PSMU. It is message reception rather than message sending. This implies that the hypothesized mechanisms of the ASMU hypothesis (reading the likes and other feedback of fellow users) are not due to ASMU but to PSMU. This conceptual confound may also explain why likes and comments cannot be dichotomized in factor analyses, as discussed in the previous section.

Based on these insights and the observed confounds in the passive-active dichotomy, future research may need to acknowledge that the interactive nature of SMU may prevent us from validly attributing potential effects of SMU to either ASMU or PSMU. The ASMU and PSMU hypotheses ignore a fundamental principle in communication theories: that there are countless psychological processes involved during message sending and reception, such as enjoyment, envy, and inspiration, which may sometimes improve well-being and/or worsen ill-being and other times dampen well-being and/or relieve ill-being.

\section{Explanation 6: heterogeneous content}

Another explanation for the unconvincing associations of ASMU and PSMU with wellbeing/ill-being may be that, without any exception, all 40 included survey-based studies focused on time spent with ASMU and/or PSMU. This also applied to the two studies that relied on log data (Bayer et al., 2018; Marengo et al., 2021), which yielded comparably weak associations of ASMU with well-being. Investigating time spent with ASMU and PSMU may be too coarse to yield significant effects on well-being or ill-being. Instead, studies should recognize that both ASMU and PSMU may cover a range of different types of content, which may yield different effects on well-being and ill-being. For example, it is likely that uplifting SM messages (e.g. humor, pictures of puppies) lead to 
increases in well-being, whereas disturbing messages (e.g. violent or discriminating content) may have negative effects on well-being. After all, in media effects research content is king. And time-based measures are less suitable than content-based measures to serve this king.

However, the neglect of content is not a weakness of the specific studies included in this review, but it is an inherent weakness of any survey and ESM design. It has been shown, for example, that specific content, such as travel posts, can evoke more negative reactions than other content (Krasnova et al., 2015). But how to measure exposure to such posts in a survey or ESM study with tight space restrictions? After all, travel posts are just one type of content out of an endless array of content that may affect well-being or ill-being positively or negatively. Investigating SM content can better be realized in experimental studies, such as in Meier et al. (2020), which found that exposure to inspiring Insta travel posts led to increases in positive affect. Investigating SM content within naturalistic, survey-based studies can also be realized, but only when it is linked to additional data collection methods, such as random screenshots of SM interactions as proposed in the "Screenomics" approach (Reeves et al., 2021), mobile sensing (Harari et al., 2020), or via SM data downloads (Boeschoten et al., 2020). Such combined data sources are another important avenue for future survey-based research.

\section{Explanation 7: heterogeneous reception}

A final viable explanation for the limited support for both the ASMU and PSMU hypotheses may lie in the heterogeneous reception of positive and negative ASMU and PSMU across SM users. It is well possible that the associations found in this review were weak and inconsistent because they were diluted across heterogeneous samples of respondents with different susceptibilities to the effects of SMU. Media effects theories agree that the unique experiences of individuals in the (social) media context shape the effects of this context (Slater, 2007; Valkenburg and Peter, 2013).

Qualitative studies have repeatedly found that media users differ greatly in their responses to SM. For example, Rideout and Fox (2018) found that some adolescents with a low mood turn to humorous SM content to enhance their mood, while others with a low mood selectively avoid SMU, and for yet others, browsing positive posts of their friends worsens their moods. These diverging reactions have been confirmed in selective exposure experiments, which showed that some individuals with pre-existing low mood tend to repair this mood by turning to SM profiles of relatively unattractive and unsuccessful people (i.e. downward comparison, Johnson and Knobloch-Westerwick, 2014), whereas others with low mood turn to SM profiles of attractive and successful people, which subsequently improve their mood (Johnson and Knobloch-Westerwick, 2017).

Several recent ESM studies adopting a person-specific effects approach have provided first evidence for the hypothesis that heterogenous reception may explain the weak and inconsistent effects of ASMU and PSMU on well-being. For example, Beyens et al. (2020) found that the PSMU hypothesis applied to only $10 \%$ of adolescents. In a subsequent study, Beyens et al. (2021) found that among 11\% of adolescents, both ASMU and PSMU led to negative effects on affective well-being. Conversely, among $12 \%$ of adolescents both ASMU and PSMU led to positive effects on well-being. Finally, Valkenburg 
et al. (2022) found that the PSMU hypothesis was confirmed for $20 \%$ of adolescents and rejected for $80 \%$, again suggesting that different persons respond to SM differently.

Together, these qualitative, selective exposure, and person-specific media effects studies indicate that when individuals are susceptible to experience negative effects of SMU, they may experience such effects due to both ASMU and PSMU. These studies also suggest that person-specific susceptibilities to positive and negative effects of SMU may depend on situational (e.g. transient mood) or more stable dispositional as well as social-context factors, such as fear of missing out or susceptibility to peer influence. In all, if we truly want to understand the unique effects of SMU on individuals, we need designs that take both content heterogeneity and reception heterogeneity into account.

\section{Measuring ASMU and PSMU in future studies}

The included studies in this scoping review used a hodgepodge of operationalizations of ASMU and PSMU. Some studies subjected their ASMU and PSMU items to a factor analysis, which ended up in two separate factors (and subsequent scales) that both included ASMU items (e.g. Thorisdottir et al., 2019). Other studies measured ASMU, but included items that pertained to chatting, which is confounding because chatting is interactive and thus involves ASMU and PSMU (see Supplemental Appendices A and B). Yet other studies compared ASMU and PSMU but used incomparable response options for both scales (Hanna et al., 2017; Wenninger et al., 2019). And yet other studies mixed public and private forms of ASMU and PSMU in their scales, which is also problematic due to the different affordances and uses of private and public SMU.

Recently, Trifiro and Gerson (2019) offered guidance on how to create universal and valid measures of both types of SMU. However, although we do not deny that it is worthwhile to investigate the potential effects of browsing, posting, interacting, or other types of SMU on well-/ill-being, our results strongly suggest that it is no longer fruitful to investigate the crude hypotheses that time spent on ASMU leads to positive effects and time spent on PSMU to negative effects. Such studies do not help us understand the potential positive and negative effects of SMU on well-being, ill-being, or any other outcome. It is time to focus on more nuanced measures of SMU, which take characteristics of SM content (e.g. its valence, intimacy, or privateness/publicness), its senders (e.g. pre-existing mood, motivations), and receivers (e.g. person-specific susceptibilities) into account. Only such measures may help us understand why, when, and for whom SMU can lead to positive or negative effects on well-being and/or ill-being.

\section{Acknowledgements}

The authors thank Wieneke Rollman for her contribution to the literature search and coding for this study.

\section{Author contributions}

Patti Valkenburg: Study conceptualization, literature search, coding of studies, and writing and revising drafts and supplemental appendices; Irene van Driel: Literature search, coding of studies, creating and editing supplemental appendices, commenting and editing drafts; Ine Beyens: Commenting and editing drafts. 


\section{Declaration of conflicting interests}

The authors declared no potential conflicts of interest with respect to the research, authorship, and/ or publication of this article.

\section{Funding}

The author(s) disclosed receipt of the following financial support for the research, authorship, and/ or publication of this article: This study was funded by an NWO Spinoza Prize and a Gravitation grant (NWO Grant 024.001.003; Consortium on Individual Development) awarded to Patti Valkenburg by the Dutch Research Council (NWO).

\section{ORCID iDs}

Patti M Valkenburg (iD https://orcid.org/0000-0003-0477-8429

Ine Beyens (iD https://orcid.org/0000-0001-7023-867X

\section{Supplemental material}

Supplemental material for this article is available online.

\section{References}

Aalbers G, McNally RJ, Heeren A, et al. (2019) Social media and depression symptoms: a network perspective. Journal of Experimental Psychology 148(8): 1454-1462.

Adachi P and Willoughby T (2015) Interpreting effect sizes when controlling for stability effects in longitudinal autoregressive models: implications for psychological science. European Journal of Developmental Psychology 12(1): 116-128.

Alfasi Y (2019) The grass is always greener on my friends' profiles: the effect of Facebook social comparison on state self-esteem and depression. Personality and Individual Differences 147: 111-117.

Bayer JB, Ellison N, Schoenebeck S, et al. (2018) Facebook in context(s): measuring emotional responses across time and space. New Media \& Society 20(3): 1047-1067.

Bayer JB, Triệu P and Ellison NB (2020) Social media elements, ecologies, and effects. Annual Review of Psychology 71: 471-497.

Bazarova NN, Choi YH, Sosik VS, et al. (2015) Social sharing of emotions on Facebook: channel differences, satisfaction, and replies. In: Proceedings of the 18th ACM conference on computer supported cooperative work \& social computing, 28 February 2015, pp. 154-164. New York: ACM Digital Library.

Beyens I, Pouwels JL, van Driel II, et al. (2020) The effect of social media on well-being differs from adolescent to adolescent. Scientific Reports 10: 10763.

Beyens I, Pouwels JL, van Driel II, et al. (2021) Social media use and adolescents' well-being: developing a typology of person-specific effect patterns. Communication Research.

Boeschoten L, Ausloos J, Moeller J, et al. (2020) Digital trace data collection through data donation. Arxiv Preprint Arxiv: 201109851.

Brailovskaia J and Margraf J (2019) I present myself and have a lot of Facebook-friends: am I a happy narcissist!? Personality and Individual Differences 148: 11-16.

Burke M, Marlow C and Lento T (2010) Social network activity and social well-being. In: Proceedings of the SIGCHI Conference on human factors in computing systems, Atlanta, GA, p. 1909-1912. 
Burnell K, George MJ and Underwood MK (2020) Browsing different Instagram profiles and associations with psychological well-being. Frontiers in Human Dynamics 2: 6.

Carr CT, Wohn DY and Hayes RA (2016) As social support: relational closeness, automaticity, and interpreting social support from paralinguistic digital affordances in social media. Computers in Human Behavior 62: 385-393.

Chen S, Shao B-J and Zhi K-Y (2019) Examining the effects of passive WeChat use in China. International Journal of Human-Computer Interaction 35(17): 1630-1644.

Choi S and Kim E-M (2021) Between Instagram browsing and subjective well-being: social comparison or emotional contagion? Media Psychology 24: 866-890.

Deters FG and Mehl MR (2012) Does posting Facebook status updates increase or decrease loneliness? An online social networking experiment. Social Psychological and Personality Science 4(5): 579-586.

Diener E (1984) Subjective well-being. Psychological Bulletin 95(3): 542-575.

Erliksson OJ, Lindner P and Mörtberg E (2020) Measuring associations between social anxiety and use of different types of social media using the Swedish Social Anxiety Scale for Social Media Users: a psychometric evaluation and cross-sectional study. Scandinavian Journal of Psychology 61(6): 819-826.

Escobar-Viera CG, Shensa A, Bowman ND, et al. (2018) Passive and active social media use and depressive symptoms among United States adults. Cyberpsychology, Behavior, and Social Networking 21(7): 437-443.

Faelens L, Hoorelbeke K, Fried E, et al. (2019) Negative influences of Facebook use through the lens of network analysis. Computers in Human Behavior 96: 13-22.

Frison E and Eggermont S (2020) Toward an integrated and differential approach to the relationships between loneliness, different types of Facebook use, and adolescents' depressed mood. Communication Research 47(5): 701-728.

Gignac GE and Szodorai ET (2016) Effect size guidelines for individual differences researchers. Personality and Individual Differences 102: 74-78.

Greenspoon PJ and Saklofske DH (2001) Toward an integration of subjective well-being and psychopathology. Social Indicators Research 54(1): 81-108.

Hancock JT, Liu SX, French M, et al. (2019) Social media use and psychological well-being: a meta-analysis. In: 69th Annual International Communication Association conference (ICA), Washington, DC, 24-28 May.

Hanley SM, Watt SE and Coventry W (2019) Taking a break: the effect of taking a vacation from Facebook and Instagram on subjective well-being. PLoS One 14(6): e0217743.

Hanna E, Ward LM, Seabrook RC, et al. (2017) Contributions of social comparison and selfobjectification in mediating associations between Facebook use and emergent adults' psychological well-being. Cyberpsychology Behavior and Social Networking 20(3): 172-179.

Harari GM, Müller SR and Gosling SD (2020) Naturalistic assessment of situations using mobile sensing methods. In:Rauthmann JF, Sherman RA and Funder DC (eds) The Oxford Handbook of Psychological Situations. Oxford: Oxford University Press, p. 299311.

Jarman HK, Marques MD, McLean SA, et al. (2021) Motivations for social media use: associations with social media engagement and body satisfaction and well-being among adolescents. Journal of Youth and Adolescence 50(12): 2279-2293.

Johnson BK and Knobloch-Westerwick S (2014) Glancing up or down: mood management and selective social comparisons on social networking sites. Computers in Human Behavior 41: 33-39.

Johnson BK and Knobloch-Westerwick S (2017) When misery avoids company: selective social comparisons to photographic online profile. Human Communication Research 43(1): 54-75. 
Keles B, McCrae N and Grealish A (2020) A systematic review: the influence of social media on depression, anxiety and psychological distress in adolescents. International Journal of Adolescence and Youth 25(1): 79-93.

Kim S, Favotto L, Halladay J, et al. (2020) Differential associations between passive and active forms of screen time and adolescent mood and anxiety disorders. Social Psychiatry and Psychiatric Epidemiology 55(11): 1469-1478.

Krasnova H, Widjaja T, Buxmann P, et al. (2015) Why following friends can hurt you: an exploratory investigation of the effects of envy on social networking sites among college-age users. Information Systems Research 26(3): 585-605.

Kross E, Verduyn P, Sheppes G, et al. (2021) Social media and well-being: pitfalls, progress, and next steps. Trends in Cognitive Sciences 25(1): 55-66.

Lian S-L, Sun X-J, Yang X-J, et al. (2020) The effect of adolescents' active social networking site use on life satisfaction: the sequential mediating roles of positive feedback and relational certainty. Current Psychology 39(6): 2087-2095.

Lin S, Liu D, Liu W, et al. (2021) Mediating effects of self-concept clarity on the relationship between passive social network sites use and subjective well-being. Current Psychology 40(3): 1348-1355.

Liu D, Baumeister RF, Yang C-C, et al (2019) Digital communication media use and psychological well-being: a meta-analysis. Journal of Computer-Mediated Communication 24: 259-274.

Marengo D, Montag C, Sindermann C, et al. (2021) Examining the links between active Facebook use, received likes, self-esteem and happiness: a study using objective social media data. Telematics and Informatics 58: 101523.

Masciantonio A, Bourguignon D, Bouchat P, et al. (2021) Don't put all social network sites in one basket: Facebook, Instagram, Twitter, TikTok, and their relations with well-being during the COVID-19 pandemic. PLoS One 16(3): e0248384.

Meier A and Reinecke L (2020) Computer-mediated communication, social media, and mental health: a conceptual and empirical meta-review. Communication Research 48(8): 1182-1209.

Meier A, Gilbert A, Börner S, et al. (2020) Instagram inspiration: how upward comparison on social network sites can contribute to well-being. Journal of Communication 70(5): 721-743.

Melsen WG, Bootsma MCJ, Rovers MM, et al. (2014) The effects of clinical and statistical heterogeneity on the predictive values of results from meta-analyses. Clinical Microbiology and Infection 20(2): 123-129.

Nisar TM, Prabhakar G, Ilavarasan PV, et al. (2019) Facebook usage and mental health: an empirical study of role of non-directional social comparisons in the UK. International Journal of Information Management 48: 53-62.

Pang H (2021) Unraveling the influence of passive and active WeChat interactions on upward social comparison and negative psychological consequences among university students. Telematics and Informatics 57: 101510.

Peters MDJ, Godfrey CM, Khalil H, et al. (2015) Guidance for conducting systematic scoping reviews. International Journal of Evidence-based Healthcare 13(3): 141-146.

Puukko K, Hietajärvi L, Maksniemi E, et al. (2020) Social media use and depressive symptoms: a longitudinal study from early to late adolescence. International Journal of Environmental Research and Public Health 17(16): 5921.

Reeves B, Ram N, Robinson TN, et al. (2021) Screenomics: a framework to capture and analyze personal life experiences and the ways that technology shapes them. Human-Computer Interaction 36(2): 150-201.

Rideout V and Fox S (2018) Digital Health Practices, Social Media Use, and Mental Well-being among Teens and Young Adults in the US. San-Francisco, CA: HopeLab. 
Rodriguez M, Aalbers G and McNally RJ (2021) Idiographic network models of social media use and depression symptoms. Cognitive Therapy and Research.

Ryff CD, Dienberg Love G, Urry HL, et al. (2006) Psychological well-being and ill-being: do they have distinct or mirrored biological correlates? Psychotherapy and Psychosomatics 75(2): $85-95$.

Slater MD (2007) Reinforcing spirals: the mutual influence of media selectivity and media effects and their impact on individual behavior and social identity. Communication Theory 17(3): 281-303.

Thorisdottir IE, Sigurvinsdottir R, Asgeirsdottir BB, et al. (2019) Active and passive social media use and symptoms of anxiety and depressed mood among Icelandic adolescents. Cyberpsychology, Behavior, and Social Networking 22(8): 535-542.

Tosun LP and Kaşdarma E (2020) Passive Facebook Use and Depression: A Study of the Roles of Upward Comparisons, Emotions, and Friendship Type. Germany: Hogrefe Publishing, pp. 165-175.

Trifiro BM and Gerson J (2019) Social media usage patterns: research note regarding the lack of universal validated measures for active and passive use. Social Media + Society 5(2).

Valkenburg PM (2022) Social media use and well-being: what we know and what we need to know. Current Opinion in Psychology, 47.

Valkenburg PM and Peter J (2013) The differential susceptibility to media effects model. Journal of Communication 63(2): 221-243.

Valkenburg PM, Beyens I, Pouwels JL, et al. (2022) Social media browsing and adolescent wellbeing: challenging the "passive social media use hypothesis." Journal of Computer-Mediated Communication 27(1): zmab015.

Valkenburg PM, Peter J and Walther JB (2016) Media effects: theory and research. Annual Review of Psychology 67: 315-338.

van Driel II, Pouwels JL, Beyens I, et al. (2019) Posting, Scrolling, Chatting, and Snapping: Youth (14-15) and Social Media in 2019. Center for Research on Children, Adolescents, and the Media (CcaM). Amsterdam, the Netherlands.

Verduyn P, Gugushvili N, Massar K, et al. (2020) Social comparison on social networking sites. Current Opinion in Psychology 36: 32-37.

Verduyn P, Lee DS, Park J, et al. (2015) Passive Facebook usage undermines affective well-being: experimental and longitudinal evidence. Journal of Experimental Psychology 144: 480-488.

Verduyn P, Ybarra O, Résibois M, et al. (2017) Do social network sites enhance or undermine subjective well-being? A critical review. Social Issues and Policy Review 11(1): 274-302.

Wang H-Z, Yang T-T, Gaskin J, et al. (2019) The longitudinal association between passive social networking site usage and depressive symptoms: the mediating role of envy and moderating role of life satisfaction. Journal of Social and Clinical Psychology 38(3): 181-199.

Wang J-L, Gaskin J, Rost DH, et al. (2018) The reciprocal relationship between passive social networking site (SNS) usage and users' subjective well-being. Social Science Computer Review 36(5): 511-522.

Waterloo SF, Baumgartner SE, Peter J, et al. (2018) Norms of online expressions of emotion: comparing Facebook, Twitter, Instagram, and WhatsApp. New Media \& Society 20(5): 18131831.

Wenninger H, Krasnova H and Buxmann P (2014) Activity matters: investigating the influence of Facebook on life satisfaction of teenage users. Proceedings of the European conference on information systems (ECIS), Tel Aviv, Israel, 9-11 June.

Wenninger H, Krasnova $\mathrm{H}$ and Buxmann P (2019) Understanding the role of social networking sites in the subjective well-being of users: a diary study. European Journal of Information Systems 28(2): 126-148. 
Yang H (2020) Do SNSs really make us happy? The effects of writing and reading via SNSs on subjective well-being. Telematics and Informatics 50: 101384.

Yin X-Q, De Vries DA, Gentile DA, et al. (2019) Cultural background and measurement of usage moderate the association between social networking sites (SNSs) usage and mental health: a meta-analysis. Social Science Computer Review 37(5): 631-648.

Yuen EK, Koterba EA, Stasio MJ, et al. (2019) The effects of Facebook on mood in emerging adults. Psychology of Popular Media Culture 8(3): 198-206.

Zhang X-X, Rost DH, Wang J-L, et al. (2020) Active and passive social networking sites usage and negative emotions: a reciprocal relationship? Journal of Social and Clinical Psychology 39(3): 195-213.

\section{Author biographies}

Patti M Valkenburg is a University Distinguished Professor at the University of Amsterdam. Her research interests include the cognitive, emotional, and social effects of media and technologies on children, adolescents, and adults.

Irene I van Driel is a postdoctoral researcher and teacher at the Amsterdam School of Communication Research (ASCoR) at the University of Amsterdam. She is broadly interested in the mechanisms involved in the effects of media use on development.

Ine Beyens is an Assistant Professor in the Amsterdam School of Communication Research (ASCoR) at the University of Amsterdam. Her research focuses on how screen media affect the development of children and adolescents, with a special focus on how parents monitor and manage their children's responses to media. 